\title{
Study of a additive device which measures the angled walls of injection mold components on a three axis CNC
}

\author{
Bogdan Pralea* and Gheorghe Nagîţ \\ Gheorghe Asachi Technical University of Iaşi, Department of Machine Manufacturing Technology, \\ Faculty of Machine Manufacturing and Industrial Management, Bdul Mangeron, 59A, 700050, Iaşi, \\ România
}

\begin{abstract}
The paper attempts to demonstrate an efficient on machine measurement system. During this study the authors will develop a measurement device, in order to accurately analyze the dimensional precision of the angled vertical walls used to center active cavities inside an injection mold, on a three axis CNC. The designed device will work in strong connection with a touch trigger probe. The final results will show an improved accuracy of the measurement system.
\end{abstract}

\section{Introduction}

Recent studies show that the measurement probes part of CMM (Coordinate-measuring machines) represents one of the most important system of dimensional control and they are responsible for the precision of the measurements. The usage of electronic devices and modern algorithms are also improving the quality of measurements which are taken by CMMs. Some studies show that, despite the fact that they have been continuously improving and companies developed different types of probes, the most common and used one remains the triggering probes, which prevail on the industrial market, due to their lower costs and a good precision [1]. This types of probes generate a binary signal when the tip of the probe touches the surface that has to be measured. During this time the tip of the probe sends the values which can be seen and further collected. However, studies are underlining the fact that these types of probes are generating some type of errors, which can lead to damage parts in manufacturing. Some of the errors that appear could be solved by calibrating the probes, before the actual measurement takes part. In present in all types of probes a compensation of future displacement takes part [2]. During calibration stage which is done by using a master sphere with a known diameter that is placed on the tip of the probe, the effective radius is being calculated, considering various points of measuring, generating this way an average of the values for future movements [3].

Some researchers are underlining the fact that the measuring probe is the source of more than $55 \%$ of measurement errors done by a coordinate measuring machine. So in order to

\footnotetext{
* Corresponding author: bpralea@gmail.com
} 
improve the precision, devices or mathematical models can be developed. The fast and correct answers of the probe can be controlled by a computer and this opens new trends and possibilities for future generations of measurements. A large variety of types of probes is available and it is compatible with $\mathrm{CNC}$ machines and CMM machines. The dimensional inspections on the components that are manufactured and have free form surfaces, need accurate measurements on a large variety of points. That is why some papers show that planning the measurement strategy can be done by starting from the base CAD model and selecting the points which have to be measurement $[3,4]$.

Through analyses of different papers we establish that there are two types of measurement probes. The first one is represented by hard probes. This probes are available in a large variety and they continue to have a large horizon inside the field of coordinate measurement machines and devices. This types of probes are used manual, as they are best suited for medium or low requirements in precision of manufactured parts. They are easy to use, but their repeatability in precision of dimensional measurement is strongly connected to the operator's experience [4]. Due to the fact that each individual operator has different touch the measurement values will also differ and this will result in lots of errors. The second type of probes is represented by the triggering probes, which engage to measure when they touch the surface that has to be evaluated by means of dimensional control. Like stated above, this category of devices are very sensible and they conduct the actual control of dimensions trough the tip of the probe, generating an electronic impulse [5]. This moment is signaled by a LED. The capacity of this probe and the technology behind it makes it versatile and they are best suited for high precision jobs (die, molds and large series of manufacturing).

A conclusion for what was stated before and through analyses of paper regarding this particular field the CMMs are opened to a series of errors. Based on their components it can be shown a classification of errors source that affect the accuracy of the probes. First of all the mechanical components of the CMM's can produces errors due to the manufacturing of their entire assembly, such as table, carriages, guide ways, bearings and the carriages. This can involve differences in thermal expansion, stiffness and adjustment. All these marker are resulting in static, quasi-static and dynamic error. $[6,7]$.

Secondly the drive components, which are reserved for CNC operated CMM's. The axis, in this particular case are equipped with electronic units and servo control units. The errors can appear due to an uneven measuring speed, the mechanical load can also be uneven and vibrations of the entire structure could also generate them [8].

Thirdly the software that collects the data. This involves strictly the algorithms which collect the data and also calculated it.

In this study the authors will try to develop an experimental device for reducing the errors which appear in measuring the angular surfaces of, in this case, the cavity walls which centers the active parts of an injection mold. This particular surface requires a high level of accuracy. So the object of study will involve a close look on CNC operated CMM's and designing an experimental, which hopefully will eliminate or reduce the error level in measuring [9].

\section{Methodology and experimental procedure}

As it was stated before the object of the study will be, for the authors, for this stage the developing of an experimental device that will reduce the number of errors in measuring the angular surfaces, on a three axis $\mathrm{CNC}$, with the help of a triggering probe. The device will be manual and will function as an additive form to the triggering probe.

Till now there were created numerous solutions for mechanical measurement of the vertical and plane surfaces on three axis $\mathrm{CNC}$ machines, but almost none of them took into 
consideration the possibility of milling free form and angular surfaces and obtain measurement data from on machine measurement devices. The problem of creating new solutions that can work and be easy to use, appears when environment request it. Here we can mention the machines and the parts that are being manufactured together with the possibility of lower the costs. In order to put it in an adequate form, the companies that have as main domain the manufacturing of complex parts, in our case injection molds, need to developed devices for what it is in essence the job completion. These types of devices are usually designed and manufactured, in a short number, sometimes they are unique, and in order to answer indirectly to client needs. So to remain at the subject at hand, the problem of designing solutions for measuring devices which are versatile, is to answer to some major criteria. First of all the complexity level of the device, secondly the time and costs involving the financial resources that are allocated for the outlook and testing and thirdly the number of devices that are manufactured.

The final stage of designing the correct model of the experimental device, was to choose the final ideas that are based on value analysis, of some multi-criteria methods, using basic points, which start from advantages and disadvantages of proposed solutions. Another step was the identification of a simple way to use for the operator, this means in particular that it needs to be manipulated on and off the measured surface with ease, never the less the active surfaces that enter in contact with the surfaces should be smooth and easy to clean form any residual material, obtained from the actual manufacturing stage. After that the stage of material selection. In this case the authors considered the factors that have an impact for this particular experimental device. So in this way the authors have chosen a material which has good machinability properties and can have good precision in time. Another stage is represented by the dimension of the experimental device. The authors first made an evaluation of the dimensions and on which we can work on. The final dimensions needed to answer the request of most surfaces for which was designed. From previous historic data we manage to extract an angle which appears always in terms of centering the active parts of the mold. It needs to answer the give geometric tolerances due to the fact that it is an additive system which is helping to reduce errors regarding measurement. Because the dimensions of the device determine the rigidity of the part and the rigidity has a strong impact on maintaining initial position and also accuracy.

To keep the design on track, it were considered the fits of involved parts. If you take into consideration that the precision of the device has a direct impact on the action of measurement that we can assume that the fits which take place must be small or even better tight $(\mathrm{H} / \mathrm{h})$. Also the roughness for the entire device needs to be very small. Another stage will be represented by choosing different technical activities, other than previous ones, conditions that are extracted from the manufacturing stage of the experimental device, the assembly and also the way we operate with. Between this conditions, is to be mentioned the fact that the device surfaces, which make contact with the part that needs to be measured, must be grinded.
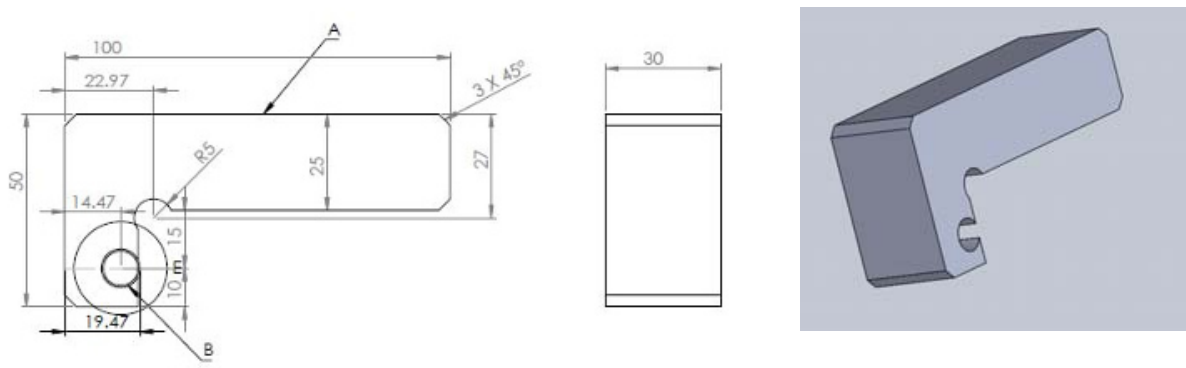

Fig. 1. Experimental device. 
Taken into consideration the facts which were discussed above and the research, the authors developed the device which is represented in Figure 1. It is characterized through a simple design and represents an additive mechanism to the triggering probe installed on the three axis CNC.

The device is an assembly, and it is composed from two parts:

- Part A represents the main body, made from 1.2312 (40CrMnNiMo8-6-4), because it is delivered in a quenched and tempered conditions at around $950-1100 \mathrm{~N} / \mathrm{mm}^{2}$, which makes it rigid and stable, also the composition makes it suitable for better machinability capacity. It was milled from two fixtures on a DOOSAN $750 \mathrm{~L}$, which is a vertical machining center which is designed for high speed and precision of small to large work pieces such as dies molds and other complex parts [10]. It operates on three axis, $\mathrm{X}$ and $\mathrm{Y}$ movement is generated by the table, $\mathrm{Z}$ axis controls the head movement. In the first fixture had several operations like roughing, pre-finishing and finishing of the outside contour. Both hole $(\Phi 10 \mathrm{~mm})$ and open cavity (R5mm) which appear in the drawing, were subject to drilling and afterwards reaming. It was very important keeping the geometric tolerances tight during milling and drilling process. At the end the part resulted according to initial specifications.

- Part B represents a shaft which was cold mounted into the main body A. The material used for this part is from a broken widia end mill, which had a diameter of $10 \mathrm{~mm}$. We cut the shank of the mill and afterwards made two chamfers, one at each end. Afterwards we inserted tightly into part A. We choose the shank of a high accuracy end mill, due to its high precision and small roughness, being the most important part of the assembly.

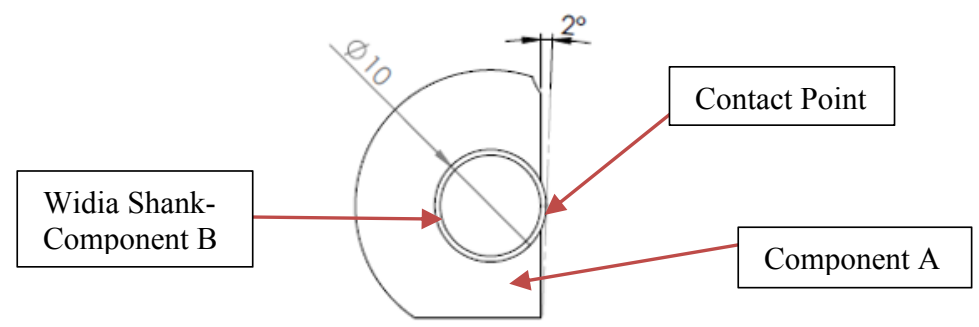

Fig. 2. Detail E from the Experimental device, represented in Figure 1.

As suggested above, in the Figure 2 is represented more clearly component $\mathrm{B}$ of the assembly, tightly fitted into component $\mathrm{A}$. The construction line on the left which has an angular value of two degrees signify the theoretical surface that needs to be measured. The surface is the one part of the cavity (Figure 3.a.) which centers the active part of the injection mold (Figure 3.b.). Both components where milled on the same three axis CNC machine as the experimental device and both are parts subject to high precision. Assuming the accurate circularity of the shaft, it is known that a circle will touch/tangent a straight line in only one point. Starting from this we can assume that putting the shank tangent with the surface will eventually generate a line which will be characterize in terms of coordinates. The coordinates will be define according to the part main origin from which the triggering probe will also start the measuring cycle.

Basically the experimental device will translate the measuring line from the angular surface with a fixed given value into a simple vertical surface which can be measured by the triggering probe, in our case the Renishaw RMP60, which is placed on the CNC machine together with a software package Inspection Plus which is generating the cycle measuring programs [11]. It has several operating cycles such as: $\mathrm{X}$ or $\mathrm{Y}$ or $\mathrm{Z}$ single surface measure, web or pocket, bore/boss, external corner, single point (radial) measurement, two- 
point (diameter) measurement. As it can be observed none of which include angular measurement of surfaces. This is where the experimental device takes places, being an additive mechanism to the entire process and assuring a lower cost with good accuracy. As it was discussed during the first part of the paper, many error that can appear and affect the accuracy of collected values is given by drive system for the CMM's that are installed on CNC's, which is the case and also the algorithm inside the software, which again is missing, part because the installed software is suited for a three axis CNC and part because that particular macro would have higher costs and the precision of the measurement will be affected by the random movement of the probe tip (sphere).

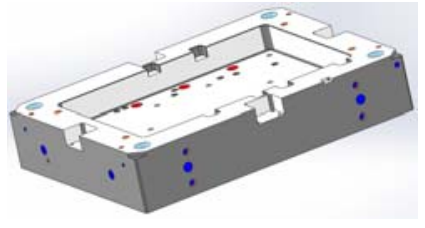

(a)

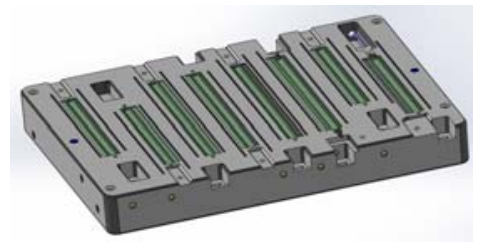

(b)

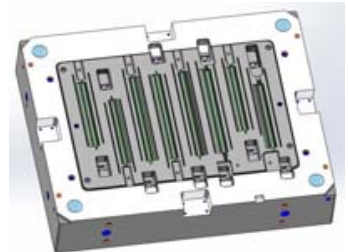

(c)

Fig. 3. Plate (a) which centers and keeps the active part of the mold (b); together assembled (c).

The process is illustrated in Figure 4 and it can be observed that the probe touches the experimental device, which has translated the angular surface into a vertical one. The value that resulted and appears on the screen of machine interface needs now to be summed or subtracted according to the direction of coordinate measurement.

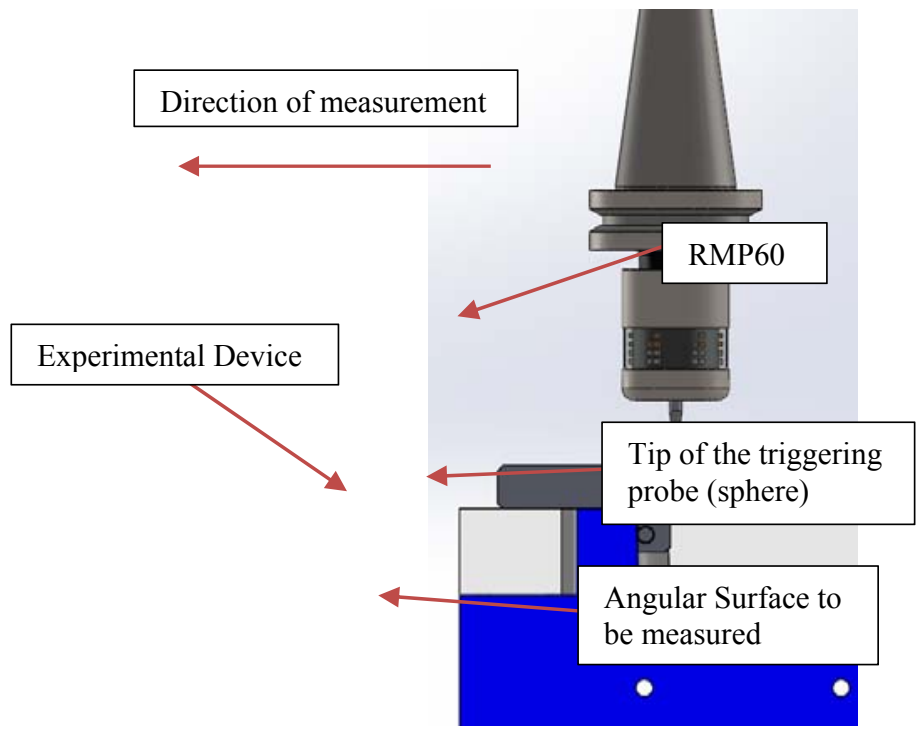

Fig. 4. The Measuring process involving the experimental device for angular surface measuring.

In order to find the value that is going to be added or subtracted we can apply the tangent in the virtual right triangle formed between the angular surface and the surface of the experimental device (Figure 5). The tangent of 2 degrees will result in a value of 0.035 and this multiply by $15 \mathrm{~mm}$ will result into a constant value $x$, given by equations number 2 , of $0.530 \mathrm{~mm}$. So if we sum that value to the known one from the vertical wall $19.47 \mathrm{~mm}$, it will give us exactly $20 \mathrm{~mm}$. That value is the one to be added or subtracted from the measured coordinate. The basic mathematical equations for this procedure are: 


$$
\begin{gathered}
|A|+x=|B| \\
x=\frac{\tan 2^{\circ}}{15}[\mathrm{~mm}]
\end{gathered}
$$

where: $|A|$-is the measured value given by the triggering probe. We will use the absolute value of it. $|B|$-represents the value of the angular surface.
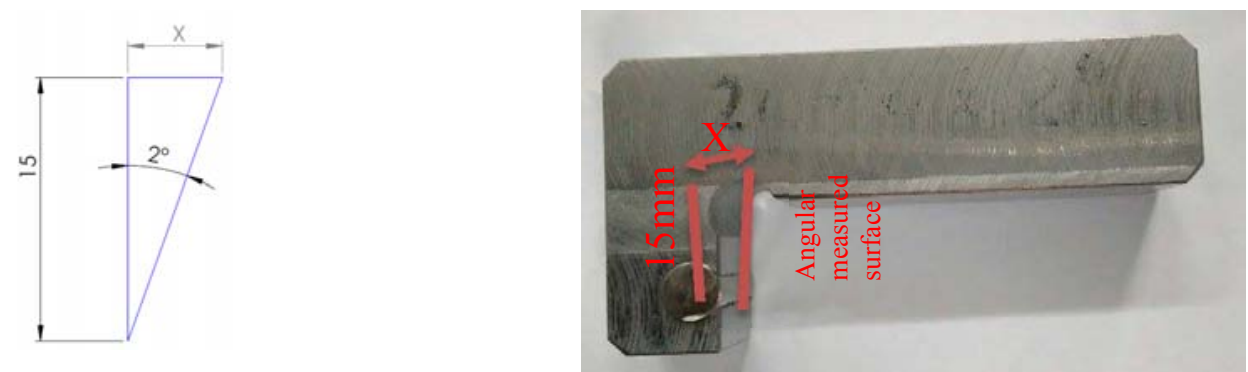

Fig. 5. The actual experimental device and the virtual right triangle that is created between it and the angular surface.

\section{Conclusions}

The present study shows that the quality and the dimensional control of the angled walls, which are machined on a three axis $\mathrm{CNC}$, are better measured using the proposed experimental device. It should also reduce the errors in measurement between the triggering probe and angular surfaces part of high precision components from injection molds.

The authors would like to thank TREVIS GRUP ROMANIA and UNITEAM ITALY, for their assistance and for having them enabled the factory equipment.

\section{References}

1. C.K. Gohel, A.H. Makwana, , Int. J. Adv. Manuf. Technol. 4, pp 21-26 (2014)

2. Abhijit Sahasrabudhe, Xiambing Liu, Theoretical and Experimental Study on Surface Finish for Multi-Axis CNC Milling, University of California, Davis CA 95916, (2003)

3. Salah H. R. Ali, Measurement Science Review 10 (2010)

4. W.G. Weekers, Compensation for dynamic errors of coordinate measuring machines, (Eindhoven University of Technology, 1996)

5. Gyula Hermann, Acta Polytechnica Hugarica 4 (2007)

6. Zhenjiu Zhang, Hong Hu, Int. J. Adv. Manuf. Technol. 69, 1483-1497 (2013)

7. H. B. Liu, Y. Q. Wang, Int. J. Adv. Manuf. Technol. 80, 1721-1731 (2015)

8. Gaiyun He, Xin Huang, Wenkui Ma, Guanmin Yu, Int. J. Adv. Manuf. Technol. doi:10.1007/s00170-016-9932-2 December (2016)

9. http://www.doerrenberg.de

10. http://www.doosanmachinetools.com/en/main.do

11. http://www.renishaw.com/en/machine-tool-probes-and-software--6073 